\title{
Dynamic functional connectivity changes in dementia with Lewy bodies and Alzheimer's disease
}

\author{
Julia Schumacher ${ }^{\mathrm{a}, *}$, Luis R. Peraza ${ }^{\mathrm{a}, \mathrm{b}}$, Michael Firbank ${ }^{\mathrm{a}}$, Alan J. Thomas ${ }^{\mathrm{a}}$, Marcus Kaiser ${ }^{\mathrm{b}, \mathrm{c}}$, \\ Peter Gallagher ${ }^{\mathrm{c}}$, John T. O'Brien ${ }^{\mathrm{d}}$, Andrew M. Blamire ${ }^{\mathrm{e}}$, John-Paul Taylor ${ }^{\mathrm{a}}$ \\ a Institute of Neuroscience, Newcastle University, Campus for Ageing and Vitality, Newcastle upon Tyne NE4 5PL, United Kingdom \\ ${ }^{\mathrm{b}}$ Interdisciplinary Computing and Complex BioSystems (ICOS) research group, School of Computing, Newcastle University, Newcastle upon Tyne NE4 5TG, United \\ Kingdom \\ ${ }^{\mathrm{c}}$ Institute of Neuroscience, Newcastle University, The Henry Wellcome Building, Newcastle upon Tyne NE2 4HH, United Kingdom \\ ${ }^{\mathrm{d}}$ Department of Psychiatry, University of Cambridge School of Medicine, Cambridge CB2 OSP, United Kingdom \\ ${ }^{\mathrm{e}}$ Institute of Cellular Medicine \& Newcastle Magnetic Resonance Centre, Campus for Ageing and Vitality, Newcastle upon Tyne NE4 5PL, United Kingdom
}

\section{A R T I C L E I N F O}

\section{Keywords:}

Resting state fMRI

Dual regression

Sliding window

Dynamic network analysis

Neurodegeneration

Cognitive fluctuations

\begin{abstract}
A B S T R A C T
We studied the dynamic functional connectivity profile of dementia with Lewy bodies (DLB) and Alzheimer's disease $(\mathrm{AD})$ compared to controls, how it differs between the two dementia subtypes, and a possible relation between dynamic connectivity alterations and temporally transient clinical symptoms in DLB.

Resting state fMRI data from 31 DLB, 29 AD, and 31 healthy control participants were analyzed using dual regression to determine between-network functional connectivity. Subsequently, we used a sliding window approach followed by k-means clustering and dynamic network analyses to study dynamic functional connectivity. Dynamic connectivity measures that showed significant group differences were tested for correlations with clinical symptom severity.

Our results show that $\mathrm{AD}$ and $\mathrm{DLB}$ patients spent more time than controls in sparse connectivity configurations with absence of strong positive and negative connections and a relative isolation of motor networks from other networks. Additionally, DLB patients spent less time in a more strongly connected state and the variability of global brain network efficiency was reduced in DLB compared to controls. There were no significant correlations between dynamic connectivity measures and clinical symptom severity.

An inability to switch out of states of low inter-network connectivity into more highly and specifically connected network configurations might be related to the presence of dementia in general as it was observed in both $\mathrm{AD}$ and DLB. In contrast, the loss of global efficiency variability in DLB might indicate the presence of an abnormally rigid brain network and the lack of economical dynamics, factors which could contribute to cognitive slowing and an inability to respond appropriately to situational demands.
\end{abstract}

\section{Introduction}

Resting state functional MRI has been used to study changes in functional connectivity associated with different forms of dementia such as dementia with Lewy bodies (DLB) and Alzheimer's disease (AD) (Kenny et al., 2012; Lowther et al., 2014; Peraza et al., 2014; Schumacher et al., 2018). To date, most functional connectivity studies have focused on analyzing mean connectivity over the duration of a scan of several minutes, thereby implicitly assuming that functional connectivity remains stationary during that time. However, it has recently been shown that functional connectivity can vary substantially in both strength and directionality on a timescale of seconds to minutes (Chang and Glover, 2010; Hutchison et al., 2013b) and that studying these dynamics can provide important complementary information to the traditional analysis of stationary functional connectivity (Calhoun et al., 2014; Hutchison et al., 2013a). DLB is characterized by transient changes in cognition. A large number of patients experience fluctuations in cognition and attention/arousal which are qualitatively different from the less frequently seen fluctuations in other dementias, such as AD (Bradshaw et al., 2004). In DLB, they occur mostly spontaneously without any situational explanation and result in pronounced variation in cognitive ability over time (Bradshaw et al., 2004; McKeith

\footnotetext{
*Corresponding author at: Biomedical Research Building 3rd floor, Campus for Ageing and Vitality, Institute of Neuroscience, Newcastle University, NE4 5PL Newcastle upon Tyne, United Kingdom.

E-mail address: j.a.schumacher2@newcastle.ac.uk (J. Schumacher).
} 
et al., 2005). In addition, the majority of DLB patients present with visual hallucinations that are not constantly present, but recur over time (Aarsland et al., 2001). The transient nature of these symptoms suggests that changes in functional connectivity dynamics might play a particularly important role in DLB (Sourty et al., 2016). Furthermore, DLB patients often show marked slowing in information processing or bradyphrenia (Firbank et al., 2018) further suggesting that temporal aspects of brain function might be affected by DLB. We therefore studied dynamic functional connectivity in DLB compared to healthy controls and patients with AD. The aims of our study were to (1) identify the differential dynamic connectivity profile of DLB compared to healthy controls, (2) investigate how functional connectivity dynamics in DLB differ from AD, and (3) test a possible relation between abnormal connectivity dynamics and the severity of clinical symptoms in DLB.

\section{Materials and methods}

\subsection{Participants}

The study involved 102 participants over 60 years of age: 33 were diagnosed with probable $\mathrm{DLB}, 36$ with probable $\mathrm{AD}$, and 33 were agematched healthy controls (HCs) with no history of psychiatric or neurological illness. Participants from two contemporary and independent dementia studies conducted at one research center (Newcastle) were combined for this analysis. Both studies recruited patients from the local community-dwelling population who had been referred to old age psychiatry and neurology services. Dementia diagnoses were performed independently by two experienced old age psychiatrists; AD was diagnosed using the National Institute on Ageing-Alzheimer's Association (NIA-AA) criteria for probable AD (McKhann et al., 1984, 2011) while DLB was diagnosed based on the consensus criteria for probable DLB (McKeith et al., 2005). According to these criteria a diagnosis of probable DLB is made if at least two of the three core DLB symptoms (visual hallucinations, cognitive fluctuations, and Parkinsonism) are present or if one core symptom is present in the presence of one or more suggestive features including REM sleep behavior disorder, severe neuroleptic sensitivity, and low dopamine transporter uptake in the basal ganglia on PET or SPECT imaging (McKeith et al., 2005). The Clinician Assessment of Fluctuations (CAF) scale was administered prior to fMRI acquisition to assess the frequency and duration of cognitive fluctuations (Walker et al., 2000). The hallucinations subscale of the Neuropsychiatric Inventory (NPI) was used to assess the occurrence of visual hallucinations in terms of severity and frequency (Cummings et al., 1994). Furthermore, the Unified Parkinson's Disease Rating Scale (UPDRS) motor subscore was used for the assessment of motor symptoms in the dementia patients (Fahn and Elton, 1987).

Exclusion criteria for all participants included co-morbid severe or unstable medical illnesses and contraindications for MRI such as pacemakers, cochlear implants, and metal body clips. Written informed consent was obtained from all participants and both studies were approved by the local ethics committee.

\subsection{Data acquisition}

Imaging for both studies was performed on the same $3 \mathrm{~T}$ Philips Intera Achieva scanner. The imaging protocol was the same in both studies except for a different resolution of the structural scans. Structural images were acquired with a magnetization prepared rapid gradient echo (MPRAGE) sequence, sagittal acquisition, echo time $4.6 \mathrm{~ms}$, repetition time $8.3 \mathrm{~ms}$, inversion time $1250 \mathrm{~ms}$, flip angle $=8^{\circ}$, SENSE factor $=2$, and in-plane field of view $256 \times 256 \mathrm{~mm}^{2}$ with slice thickness $1.2 \mathrm{~mm}$, yielding a voxel size of $0.93 \times 0.93 \times 1.2 \mathrm{~mm}^{3}$ (study 1 ) and in-plane field of view $240 \times 240 \mathrm{~mm}^{2}$ with slice thickness $1.0 \mathrm{~mm}$, yielding a voxel size of $1.0 \times 1.0 \times 1.0 \mathrm{~mm}^{3}$ (study 2). Resting state scans were obtained with a gradient echo echo-planar imaging sequence with 25 contiguous axial slices, 128 volumes, anterior-posterior acquisition, in plane resolution $=2.0 \times 2.0 \mathrm{~mm}$, slice thickness $=6 \mathrm{~mm}$, repetition time $(\mathrm{TR})=3000 \mathrm{~ms}$, echo time $=40 \mathrm{~ms}$, and field of view $=260 \times 260 \mathrm{~mm}^{2}$. Participants were asked to keep their eyes open and stay awake for the duration of the resting state scan. DLB patients who were taking dopaminergic medication were scanned in the ON state.

\subsection{Preprocessing}

FEAT (FMRI Expert Analysis Tool, version 6.0) which is part of the FMRIB's software library (FSL, www.fmrib.ox.ac.uk/fsl) was used to perform motion correction with MCFLIRT, slice-timing correction, and spatial smoothing with a $6.0 \mathrm{~mm}$ full width at half maximum Gaussian kernel. Participants were excluded if the MCFLIRT motion parameters exceeded $2 \mathrm{~mm}$ translation and/or $2^{\circ}$ rotation. To ensure that there were no group differences in motion between the groups, motion was compared between the groups using the formula introduced in (Liao et al., 2010). Additionally, mean framewise displacement was compared between the groups following the approach of (Power et al., 2012).

A denoising procedure was performed with ICA-AROMA in FSL which performs single-subject independent component analysis (ICA) to remove motion components from each participant's functional data (Pruim et al., 2015). Subsequently, eroded CSF and white matter masks were estimated using FAST in FSL and the mean signal inside the mask was regressed out of each participant's cleaned functional data. Functional and structural images were co-registered using boundary based registration in FSL, and normalized to standard MNI space using Advanced Normalization Tools (Avants et al., 2011). As a final step, functional data were temporally high-pass filtered with a cut-off of $150 \mathrm{~s}$ and resampled to a resolution of $4 \times 4 \times 4 \mathrm{~mm}^{3}$.

\subsection{Analysis of resting state data}

Resting state networks (RSNs) were estimated with an independent set of 42 healthy control participants from two previous studies that were conducted on the same MR scanner using a similar imaging protocol (see Supplementary Table S1). Data from all 42 HCs were temporally concatenated and subjected to a group-ICA using FSL's MELODIC. A meta ICA approach was adopted to obtain reliable components (Biswal et al., 2010; Poppe et al., 2013) using a model order of 70 independent components which has been shown to be optimal for assessing disease-related group differences (Abou Elseoud et al., 2011). The meta ICA consisted of repeating MELODIC 25 times on randomized subsets of 30 out of the 42 independent HC participants. This was followed by a meta MELODIC on the concatenated components from all individual ICA runs. Components that showed a spatial correlation $>$ 0.6 across runs were selected for subsequent analysis (Cerliani et al., 2015). Additionally, the whole meta ICA procedure was repeated using all HC participants from the main analysis and the resulting components were compared to the ones obtained from the independent group to ensure that all selected RSNs were present in both cohorts (Schumacher et al., 2018). Meta ICA components that survived these reliability checks were visually inspected with respect to their spatial maps (Kelly et al., 2010) and 27 were identified as being of biological interest according to previous literature (Agosta et al., 2012; Beckmann et al., 2005) (Fig. 1 and Supplementary Table S2).

Subsequently, FSL-dual regression was run with all 27 identified RSNs concatenated in a single 4D image, to obtain subject-specific representations of the RSN spatial maps and associated subject-specific time courses (Fig. 2A). Results from a static connectivity analysis using dual regression have been published previously using the same data (Schumacher et al., 2018).

The subject-specific time courses resulting from dual regression were further processed in Matlab (R2016b) using functions from the 


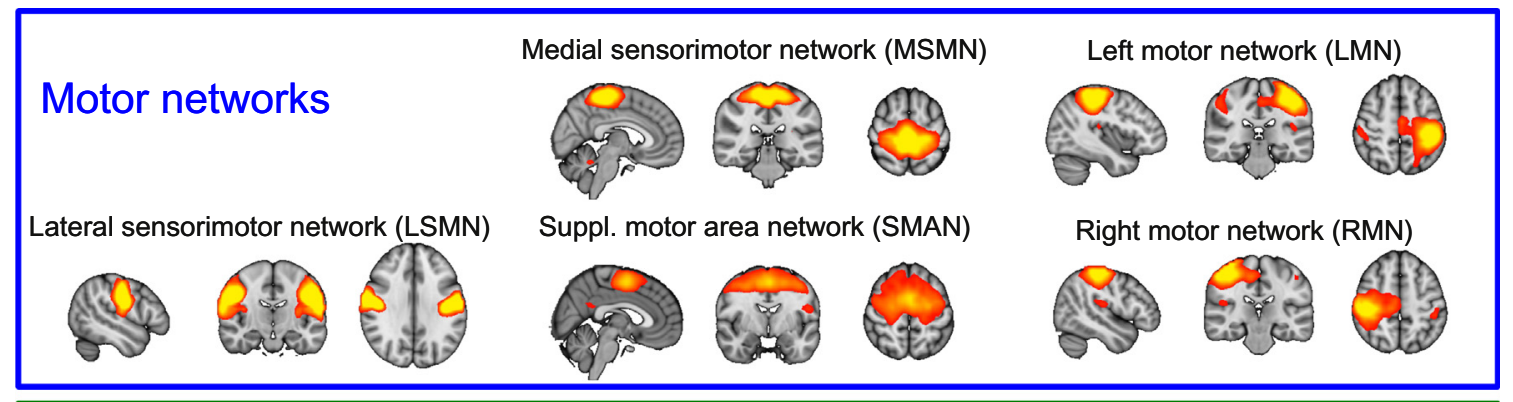

Subcortical networks

Cerebellar networks Cerebellar network 1 (CBN1)
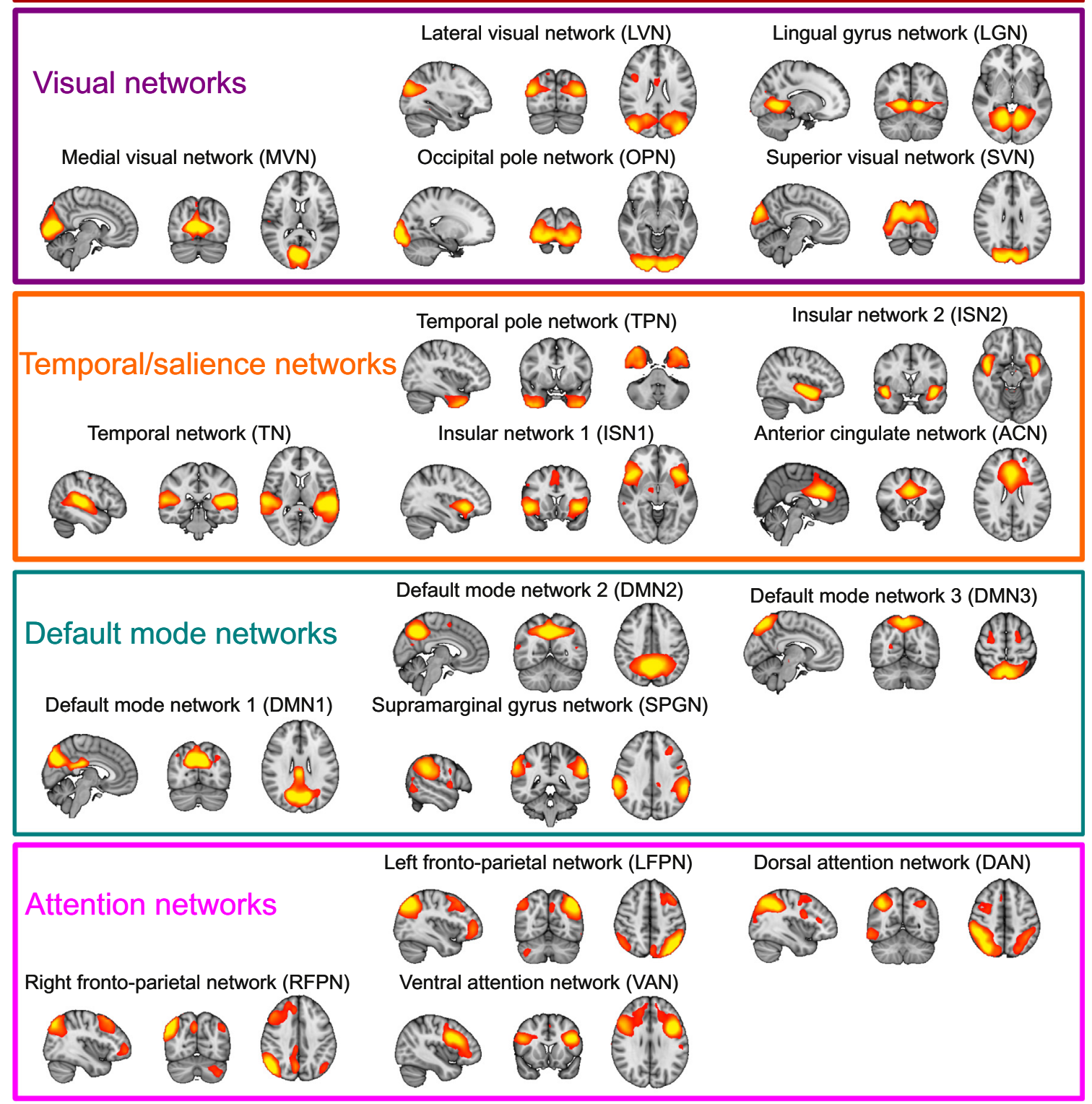

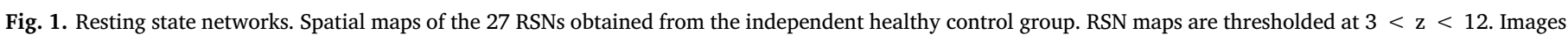
are shown in radiological convention, i.e. the left side of the image corresponds to the right hemisphere. 


\section{A) group ICA and dual regression}
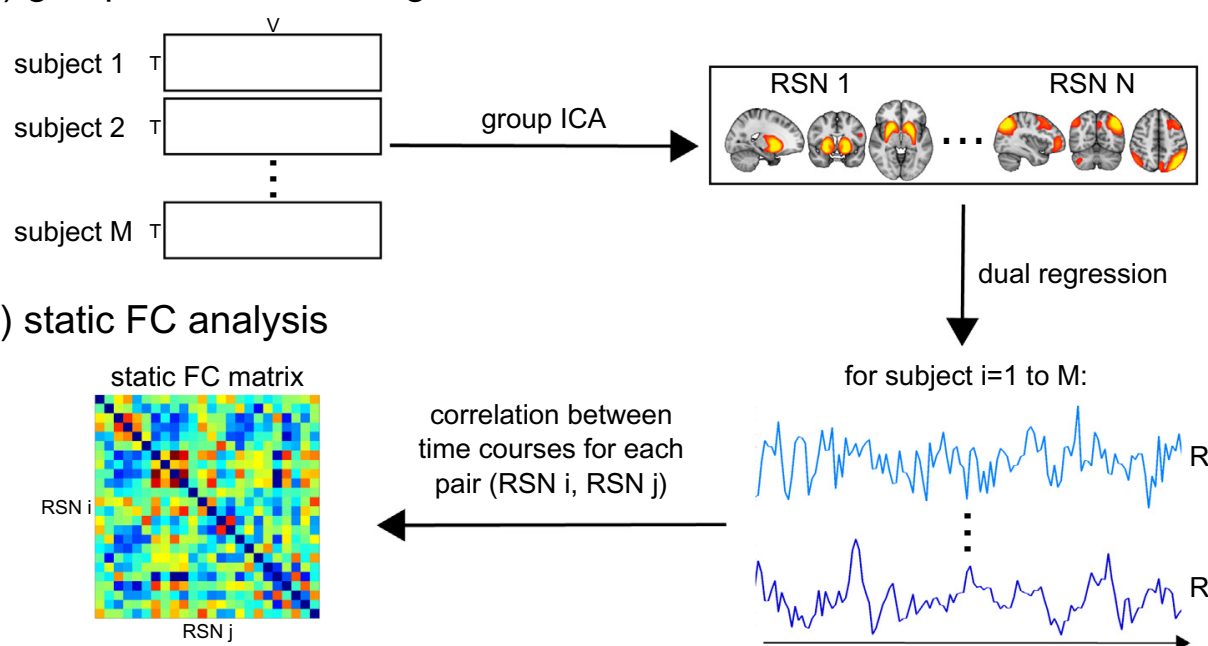

for subject $i=1$ to $M$ :

\section{C) dynamic sliding window analysis}
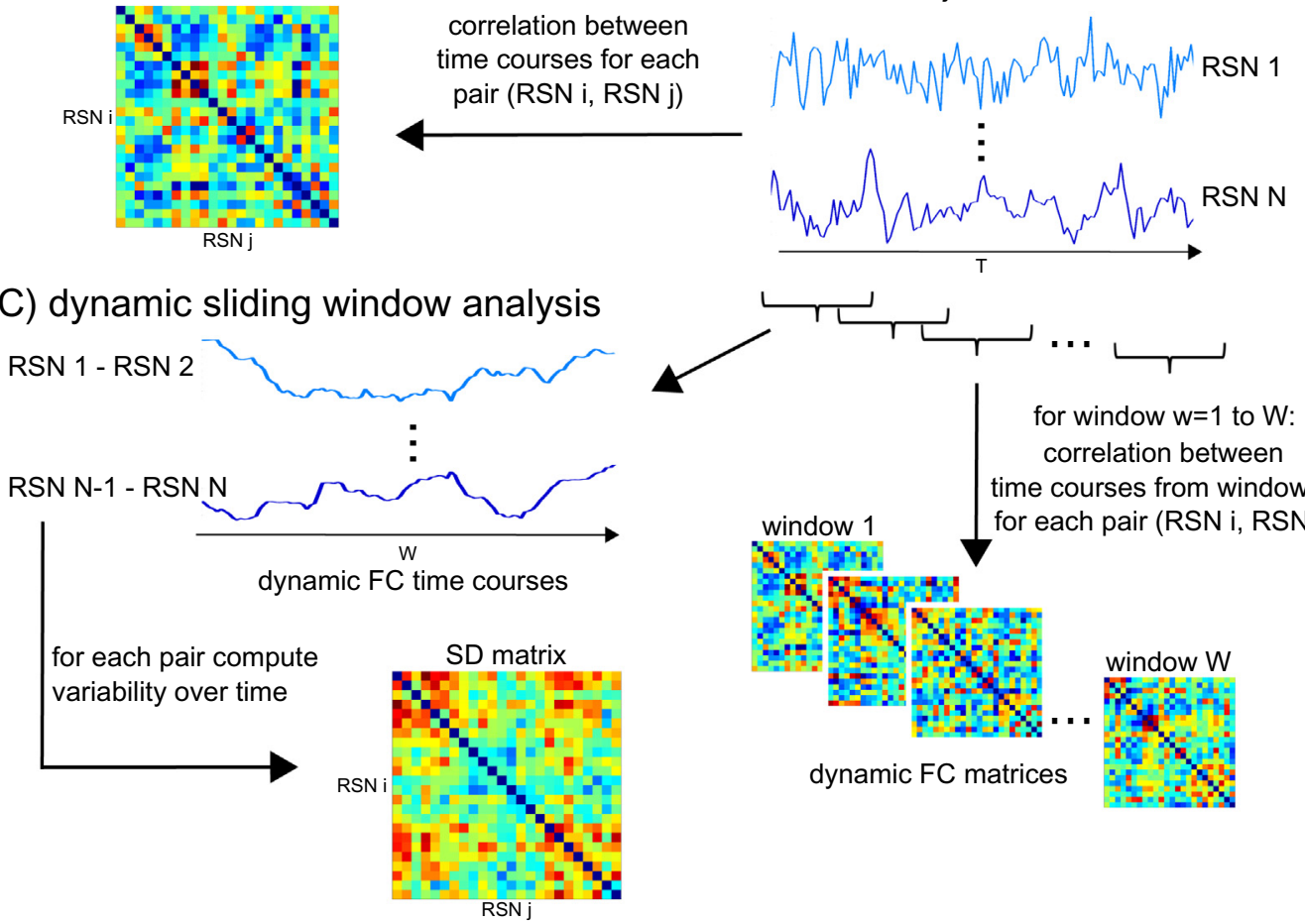

\section{D) clustering analysis}
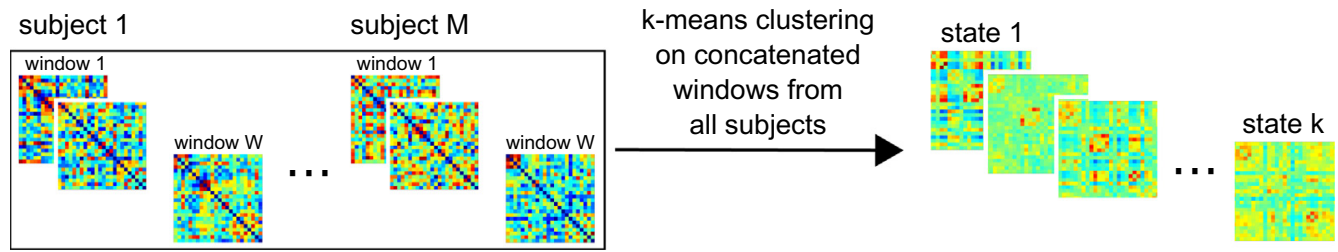

Fig. 2. Sliding window approach and k-means analysis. A) Data from all healthy control subjects from the independent cohort is concatenated in time and subjected to group ICA to identify RSN spatial maps. Subject-specific time courses of each RSN are estimated using dual regression. B) Static functional connectivity (FC) analysis by calculating correlation between each pair of RSNs using the whole time course (see (Schumacher et al., 2018)). C) Sliding window approach and estimation of standard deviation (SD) of connectivity over time. D) K-means clustering.

Group ICA of fMRI toolbox (GIFT, http://mialab.mrn.org/software/ gift/index.html) to remove remaining noise sources. Postprocessing included (1) detrending to remove linear, quadratic, and cubic trends, (2) outlier detection based on AFNI's 3dDespike function (http://afni. nimh.nih.gov/afni) and interpolation of outliers using a third-order spline fit to the clean parts of the time courses, and (3) low-pass filtering using a fifth-order Butterworth filter with a cutoff frequency of $0.15 \mathrm{~Hz}$.

\subsection{Sliding window analysis}

The postprocessed dual regression time series were analyzed with a sliding window method to assess changes in between-network connectivity over time (Fig. 2C). This analysis was performed in Matlab
(R2016b) based on functions from GIFT (Allen et al., 2014). A tapered window was created by convolving a rectangle of 22TR ( $66 \mathrm{~s}$ ) with a Gaussian with sigma of 3TR and moved in steps of 1TR. Since there were 128 volumes available, this resulted in a total of 107 overlapping time windows for each participant. To assess the robustness of the results with respect to different window sizes, all analyses were repeated for window sizes ranging from 18 to $28 \mathrm{TR}$.

A covariance matrix between all RSN-to-RSN pairs was estimated for each window separately. Following Allen et al. (2014) regularized inverse covariance matrices were estimated using the graphical LASSO approach. Regularization was applied because it has been shown that estimation of covariance based on relatively short time series can otherwise be noisy. To achieve regularization and promote sparsity, an L1-norm constraint was imposed on the inverse covariance matrix. The 
L1 regularization parameter $\lambda$ was optimized for each participant individually by evaluating the log-likelihood of unseen time windows from the same participant using 20 -fold cross-validation. All covariances were subsequently converted to correlation values and transformed into z-scores using Fisher r-to-z transformation. To control for the effect of possible covariates the z-scores were then residualized with respect to age, gender, and study membership using multiple linear regression (Damaraju et al., 2014).

The variability of the connection strengths between RSNs (dynamic functional connectivity) was assessed by calculating the standard deviation (SD) of the RSN-to-RSN correlations across time windows. To assess whole-brain dynamics the mean SD across all connections between RSN pairs was computed. Additionally, the mean SD for each network across all other networks was considered and each RSN-to-RSN connection was also tested separately.

\section{6. $K$-means clustering}

To assess patterns of functional connectivity that reoccur over time across different participants, k-means clustering was applied to the windowed covariance matrices from all windows and all participants using the Manhattan (L1) distance function (Fig. 2D). The optimal number of clusters $\mathrm{k}$ was chosen based on the elbow criterion of the cluster validity index, computed as the ratio of within-cluster to between-cluster distance (Allen et al., 2014). The clustering algorithm was repeated 500 times in Matlab with random initializations of cluster centroid positions to get a stable solution. In addition to using the optimal value for $\mathrm{k}$, the analyses were repeated for $\mathrm{k}$ ranging from 2 to 8 to assess the robustness of the results regarding different values of $k$.

Group differences were assessed with respect to (1) frequency: proportion of windows assigned to a state, (2) mean dwell time: average number of consecutive windows assigned to a state, (3) intertransition interval: average number of consecutive windows before a state transition occurs, (4) number of transitions: overall number of transitions between different states (Hutchison and Morton, 2015; Marusak et al., 2016).

\subsection{Dynamic network analysis}

We also considered a graph-theoretic approach to study the dynamics of global and local efficiency using the Brain Connectivity Toolbox (Rubinov and Sporns, 2010). For each time window a graph was constructed using the 27 RSNs as nodes and the correlation between the RSNs within the respective time window as edge strength. We created binarized, unweighted, and undirected graphs by thresholding the absolute value of the individual time window correlation matrices to achieve different edge densities. The edge density of a graph is defined as the number of existing edges divided by the maximum number of possible edges (351 in our case). We used edge density thresholds ranging from $3.7 \%$ to $39.3 \%$ based on previous network studies (Peraza et al., 2015; van Wijk et al., 2010). Global and local efficiency were computed for each time window separately (Achard and Bullmore, 2007; Latora and Marchiori, 2001). Variability of efficiency was then assessed by integrating over all edge density thresholds and computing the standard deviation of the respective measure over time (Kim et al., 2017). The same analysis was repeated in a static way by calculating local and global efficiency from the whole time course.

\subsection{Statistical analysis}

All statistical analyses were performed in R (https://www.R-project. org/). The variability of functional connectivity of each network and each connection was compared between the groups using a non-parametric multivariate ANOVA (MANOVA (Burchett et al., 2017)) with diagnosis as between-subject factor. The different k-means measures were also compared between the groups using non-parametric
MANOVA followed by Kruskal-Wallis ANOVAs and post-hoc Dunn's tests using false discovery rate (FDR) correction for multiple comparisons. Effect sizes for all group comparisons were calculated using $\mathrm{r}^{2}$ (see Supplementary Tables S3 and S5). Spearman's rank correlations between dynamic functional connectivity measures that showed significant group differences and clinical variables in the DLB group were assessed for the CAF total score as a measure of cognitive fluctuation severity, the UPDRS motor subscale as a measure of the severity of Parkinsonism, the NPI hallucination subscale which was specifically scored for the severity and frequency of visual hallucinations, and Mini Mental State Examination (MMSE) and Cambridge Cognitive Examination (CAMCOG) as measures of global cognition. In the $\mathrm{AD}$ group, correlations with MMSE and CAMCOG were calculated.

To assess the effect of dopaminergic medication on dynamic connectivity measures, DLB patients were divided into those patients who were taking dopaminergic medication and those who were not on these medications and all dynamic connectivity measures were compared between the two groups using Mann-Whitney $U$-tests (see Section 7 of the Supplementary Material).

Additionally, to investigate the possible influence of motion artefacts on the dynamic connectivity measures, we calculated correlations between mean framewise displacement and the dynamic connectivity measures across all participants (see Section 8 of the Supplementary Material).

\subsection{Effect of grey matter atrophy}

To study differences in regional grey matter (GM) volume between the three groups, a voxel-based morphometry (VBM) analysis was conducted using DARTEL in SPM12 using age, gender, and total intracranial volume as covariates. Additionally, total grey matter volume and total intracranial volume (TIV) were compared between the groups. Total GM volume and TIV were also considered as covariates when residualizing the windowed correlation matrices (see Section 2.5).

\section{Results}

One $\mathrm{AD}$ patient had to be excluded due to coregistration errors. Additionally, two controls, six AD, and two DLB participants were excluded because of excessive motion. This resulted in 31 DLB, 29 AD patients, and $31 \mathrm{HCs}$ for further analysis. The overall motion for all included participants did not differ between the groups (Kruskal-Wallis ANOVA; rotation, $\mathrm{H}_{2}=0.79, \mathrm{p}=0.67$; translation, $\mathrm{H}_{2}=0.67$, $\mathrm{p}=0.71$ ). Furthermore, mean framewise displacement was not different between the groups (HC, mean $(\mathrm{SD})=0.24(0.11)$; $\mathrm{AD}$, mean $(\mathrm{SD})=0.25$ (0.15); DLB, mean $(\mathrm{SD})=0.24$ (0.09); Kruskal-Wallis ANOVA, $\mathrm{H}_{2}=1.22, \mathrm{p}=0.54$ ).

\subsection{Demographics}

Age and gender were not significantly different between the groups and the two dementia groups did not differ significantly in terms of overall cognition (MMSE and CAMCOG) and dementia duration (Table 1). As expected, the number of patients taking dopaminergic medication was significantly higher in the DLB group while the number of patients taking cholinesterase inhibitors was not different between the dementia groups. DLB patients had worse motor function, more visual hallucinations, and greater cognitive fluctuations than $\mathrm{AD}$ patients.

\subsection{Group differences in dynamic connectivity}

The subject-specific values for the regularization parameter $\lambda$ that resulted from the optimization procedure did not differ between the three groups (Kruksal-Wallis ANOVA, $\mathrm{H}_{2}=0.06, \mathrm{p}=0.97$ ).

Fig. 3 shows matrices representing the mean SD of the strength of 
Table 1

Demographic and clinical variables, mean (standard deviation).

\begin{tabular}{|c|c|c|c|c|}
\hline & $\mathrm{HC}(\mathrm{N}=31)$ & $\mathrm{AD}(\mathrm{N}=29)$ & $\operatorname{DLB}(\mathrm{N}=31)$ & Between-group differences \\
\hline Male: female & $22: 9$ & $20: 9$ & 19:12 & $\chi^{2}=0.73, p=0.70^{a}$ \\
\hline Study 1: study 2 & $15: 16$ & $13: 16$ & $12: 19$ & $\chi^{2}=0.60, p=0.74^{a}$ \\
\hline Age & $76.4(7.2)$ & $75.2(8.6)$ & $78.1(6.7)$ & $\mathrm{F}_{2,88}=1.16, \mathrm{p}=0.32^{\mathrm{b}}$ \\
\hline AChEI & - & 26 & 28 & $\chi^{2}=0.007, p=0.93^{c}$ \\
\hline PD meds & - & 1 & 18 & $\chi^{2}=20.66, p<0.001^{c}$ \\
\hline Duration & - & $3.7(1.7)^{\mathrm{f}}$ & $3.4(2.3)$ & $\mathrm{U}=339, \mathrm{p}=0.14^{\mathrm{d}}$ \\
\hline MMSE & $28.9(1.1)$ & $21.8(3.8)$ & $22.0(4.3)$ & $\mathrm{t}_{58}=0.20, \mathrm{p}=0.85^{\mathrm{e}}$ \\
\hline CAMCOG & $96.7(3.2)$ & $70.3(13.5)$ & $73.3(13.6)$ & $\mathrm{t}_{58}=0.86, \mathrm{p}=0.39^{\mathrm{e}}$ \\
\hline UPDRS III & $1.94(2.8)$ & $3.5(4.0)$ & $18.1(10.0)$ & $\mathrm{t}_{58}=7.32, \mathrm{p}<0.001^{\mathrm{e}}$ \\
\hline CAF total & - & $1.00(2.5)^{\mathrm{f}}$ & $4.8(4.9)^{g}$ & $\mathrm{t}_{56}=3.66, \mathrm{p}=0.001^{\mathrm{e}}$ \\
\hline NPI total & - & $5.9(5.5)^{\mathrm{h}}$ & $14.6(11.0)^{\mathrm{i}}$ & $\mathrm{t}_{54}=3.68, \mathrm{p}=0.001^{\mathrm{e}}$ \\
\hline NPI hall & - & $0^{\mathrm{j}}$ & $1.6(1.8)^{\mathrm{i}}$ & $\mathrm{t}_{53}=4.53, \mathrm{p}<0.001^{\mathrm{e}}$ \\
\hline
\end{tabular}

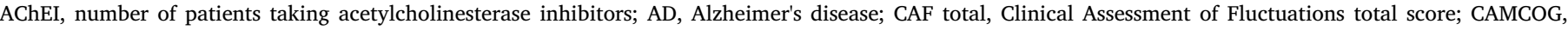

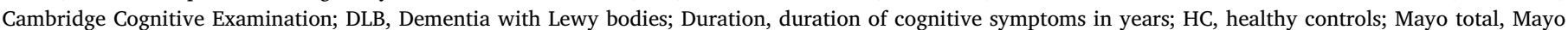

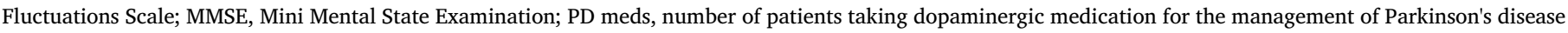
symptoms; UPDRS III, Unified Parkinson's Disease Rating Scale III (motor subsection); NPI, Neuropsychiatric Inventory; NPI hall, NPI hallucination subscore.

a Chi-square test HC, AD, DLB.

b One-way ANOVA HC, AD, DLB.

c Chi-square test AD, DLB.

d Mann Whitney $U$ test AD, DLB.

e Student's $t$-test AD, DLB.

f $\mathrm{N}=28$.

g $\mathrm{N}=30$.

h $\mathrm{N}=27$

i $\mathrm{N}=29$.

j $\mathrm{N}=26$.

each RSN-to-RSN connection within each group. Overall mean variability of connectivity is shown in the bottom right panel of Fig. 3. When considering average variability of each RSN, the overall MANOVA did not show a significant effect of diagnosis $(\mathrm{F}(10,442)=1.39, \mathrm{p}=0.18)$. Similarly, when considering each individual RSN-to-RSN connection, the MANOVA did not reveal a significant group difference across all variables $(\mathrm{F}(96,4221)=1.02, \mathrm{p}=0.43)$. Supplementary Table S3 and Supplementary Fig. S2 show effect size estimates for all comparisons. Overall, effect sizes were largest for the comparison of HC and DLB participants while they were lowest for the comparison between both dementia groups.

SD matrices were re-estimated using different window sizes from 18 to $28 \mathrm{TR}$ showing that the overall appearance of the SD matrices was not dependent upon the specific choice of window size (Supplementary Fig. S1). Furthermore, repeating the analysis of connectivity variability for different window sizes did not show any significant group differences (all $\mathrm{p}>0.05$ ).

\subsection{K-means clustering}

An optimal number of $\mathrm{k}=3$ clusters was determined by the elbow criterion (Supplementary Fig. S3). State 1 was characterized by relatively strong positive and negative between-network correlations (Fig. 4). Especially strong positive correlations were present within the visual and the motor networks and between these two groups of networks (Fig. 4E). Additionally, the motor and visual networks showed negative correlations with cognitive control, salience, and temporal networks and there was a strong connection between two components of the default mode network (DMN). In contrast, state 2 was characterized by much sparser connections, with weaker connectivity within visual and motor networks and a relative lack of connections between the two groups. There were a few positive connections between visual and default mode networks and additional positive connections between DMN and attention networks. State 2 was the most common state, being present in almost all participants and accounting for $50 \%$ of all time windows. Similar to state 2 , state 3 was characterized by weaker connections and the relative absence of strong anti- correlations. In addition to some within-module connections in the visual, motor, and default mode networks, there were weak connections between visual and DMN and attention networks.

There were no significant differences between the groups in the number of state transitions or the intertransition interval (Supplementary Tables S4 and S5). The frequency of occurrence of the three states was not correlated with time, i.e. we did not observe an increase or decrease in the occurrence of any state over the duration of the scan (Supplementary Table S4).

Non-parametric MANOVAs revealed that there was a significant effect of diagnosis on frequency and mean dwell time across all three states (Supplementary Tables S4 and S5). Follow-up univariate KruskalWallis ANOVAs and pairwise post-hoc tests demonstrated that state 1 occurred less frequently in AD and DLB compared to controls with no difference between the dementia groups (Fig. 4F and Supplementary Tables S4 and S5). In contrast, state 2 occurred more often in DLB compared to controls. However, there was no difference between HC and $\mathrm{AD}$ or between $\mathrm{AD}$ and $\mathrm{DLB}$ for state 2. The mean dwell time of state 1 and 2 followed the same pattern as the frequency, i.e. DLB patients spent shorter periods of time in state 1 and longer periods of time in state 2 than HC; AD patients spent shorter times in state 1 than HC with no difference for state 2 , and there was no difference between the dementia groups (Fig. 4G). There were no group differences in frequency or dwell time for state 3 (Supplementary Tables S4 and S5).

Several further analyses were performed to assess the robustness of the k-means analysis. Supplementary Fig. S4 shows results for different numbers of clusters demonstrating that the main result of differences in frequency and dwell time of state 1 and 2 persisted when using a higher $\mathrm{k}$. Additionally, repeating the $\mathrm{k}$-means analysis with $\mathrm{k}=3$ for different window sizes confirmed that the specific choice of window length did not influence the state identification (Supplementary Fig. S5). We also performed split-half and bootstrap resampling which showed that states 1 and 2 were consistently identified in both split-half and all bootstrap resamples, while state 3 failed to be identified in some of the bootstrap resamples (Supplementary Fig. S6). 


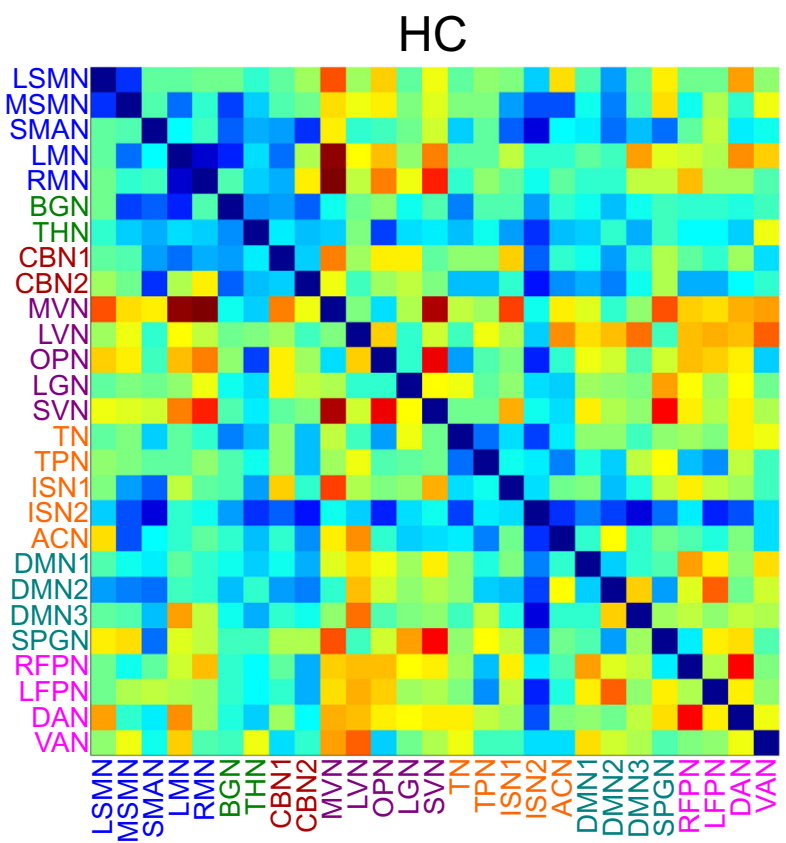

DLB

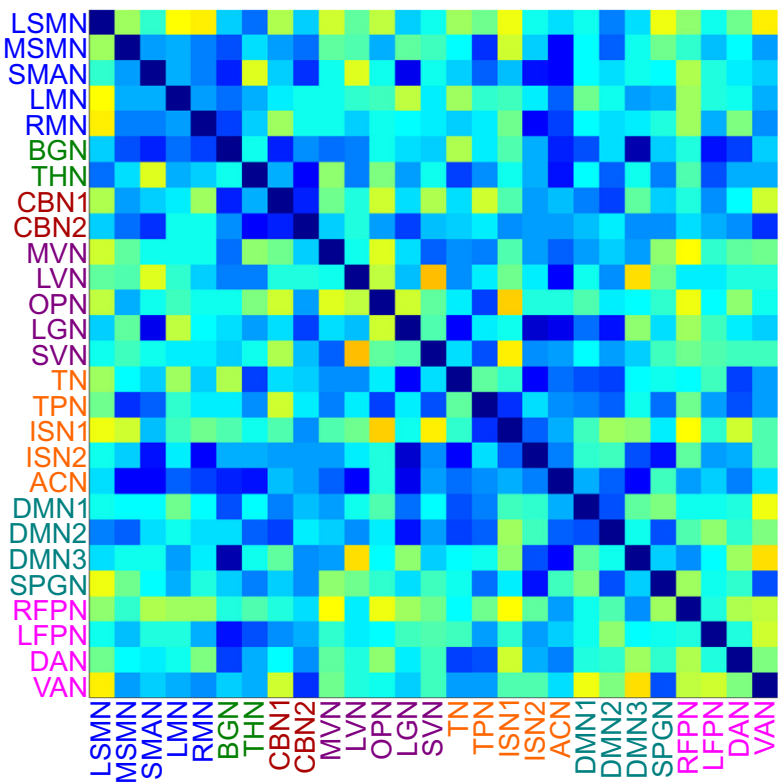

$A D$

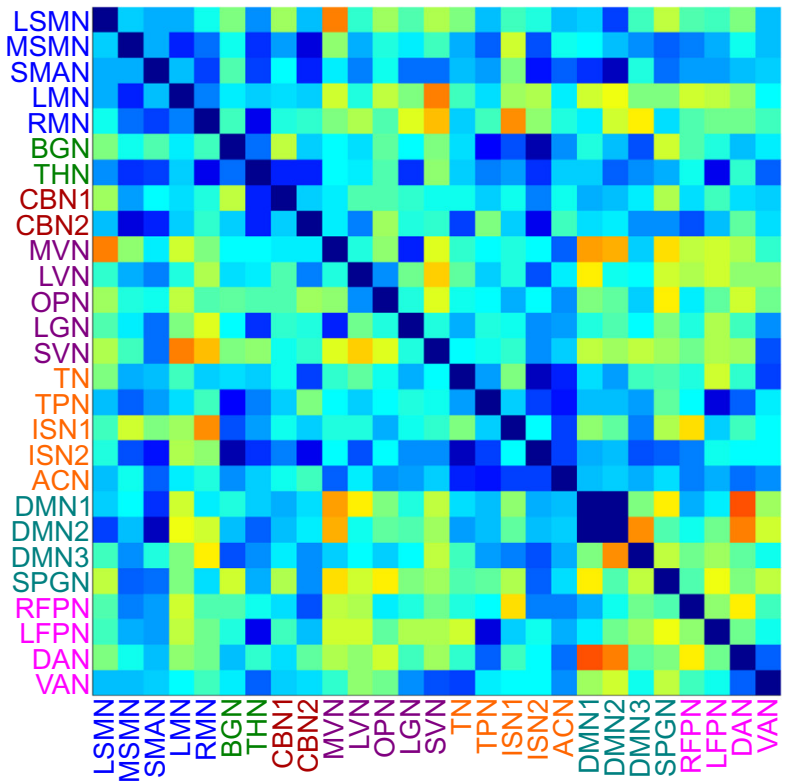

SD over time
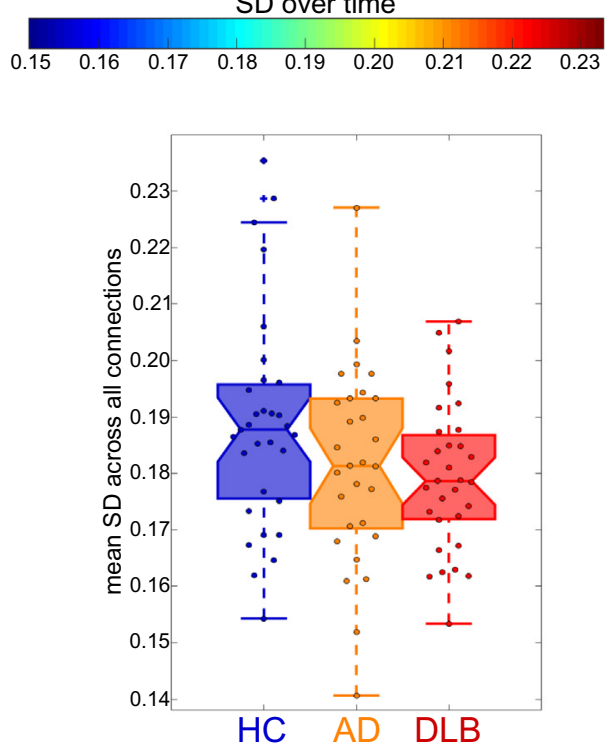

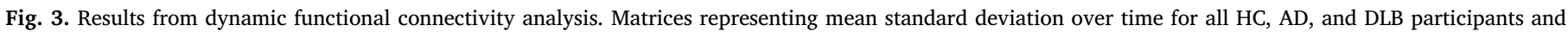
boxplot showing a group comparison of mean standard deviation across all connections.

\subsection{Dynamic network measures}

There was no difference between the groups in terms of variability of local efficiency (Fig. 5B and Supplementary Table S4). In contrast, global efficiency variability differed significantly between the groups. Post-hoc tests revealed that it was less variable in DLB compared to controls with no significant difference between $\mathrm{AD}$ and $\mathrm{HC}$ as well as between the two dementia groups (Fig. 5A and Supplementary Tables S4 and S5). Fig. 5C shows variability of global efficiency for different edge densities and indicates that the largest group difference occurred for edge densities of around $20 \%$.

The static analysis of global and local efficiency did not reveal any group differences (see Supplementary Tables S4 and S5).

\subsection{Relation to clinical scores}

Table S6 shows correlations between clinical variables and all dynamic connectivity measures that showed significant group differences in the DLB and AD group separately. There were no significant correlations between cognitive fluctuation or visual hallucination severity and the dynamic connectivity measures. Frequency of state 2 was positively correlated with the UPDRS in DLB. However, this correlation did not survive correction for multiple comparisons. There were no significant clinical correlations in the $\mathrm{AD}$ group.

Comparing DLB patients who were on dopaminergic medication to those who were not, did not reveal any significant differences between the two groups (Supplementary Table S7).

There were no significant correlations between the dynamic 


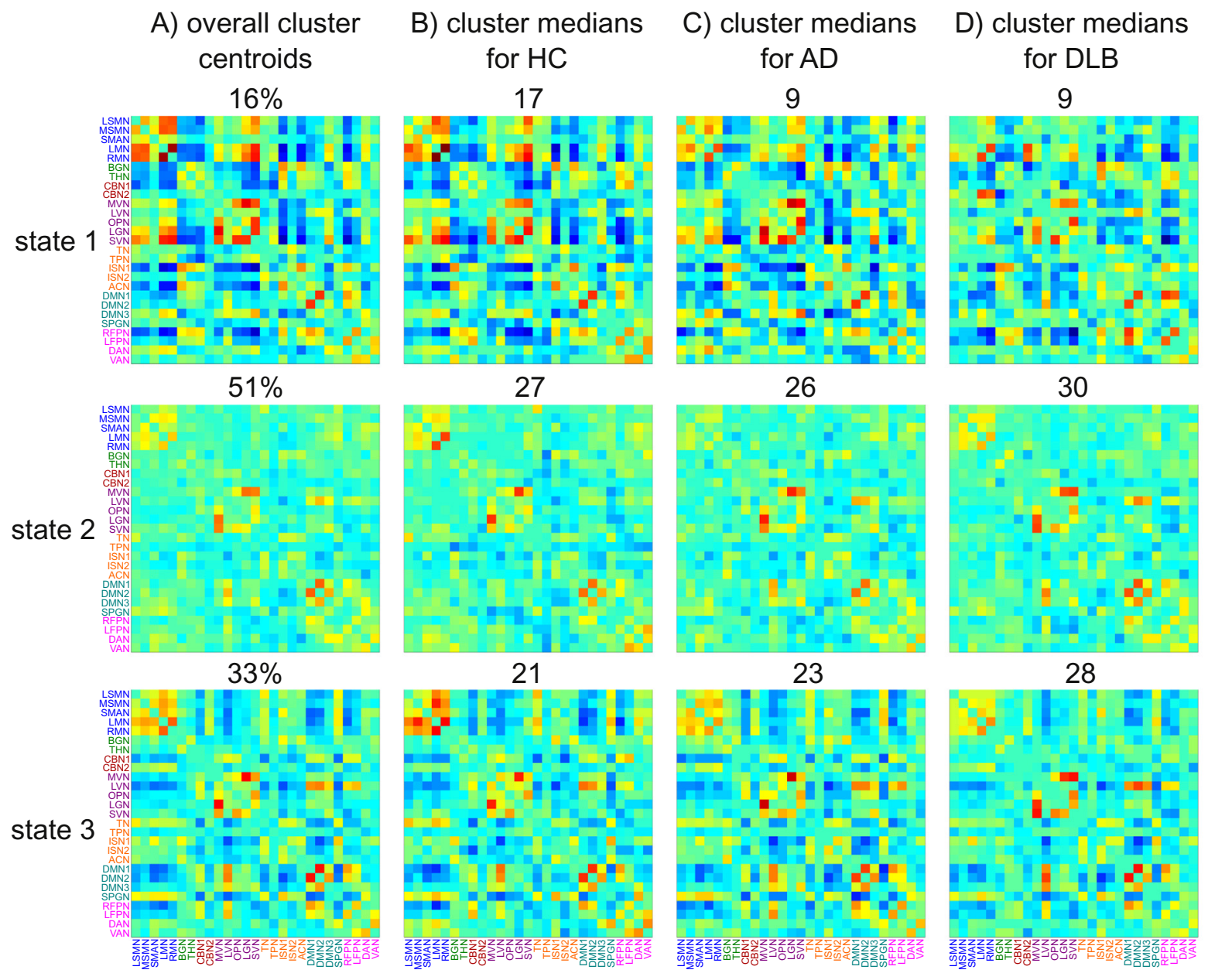

E) representation of cluster centroids
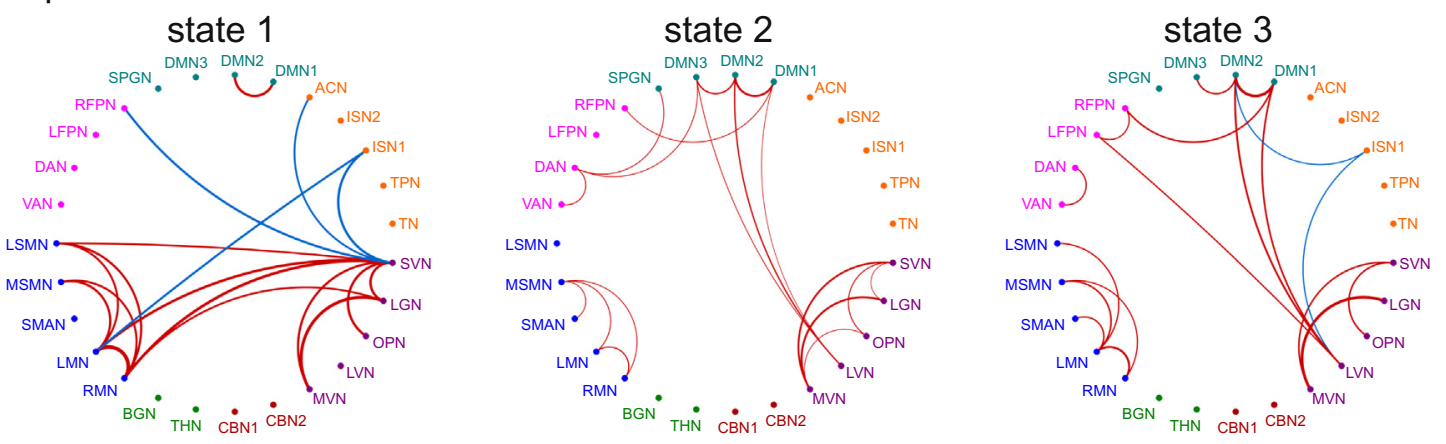

F) frequency

G) mean dwell time
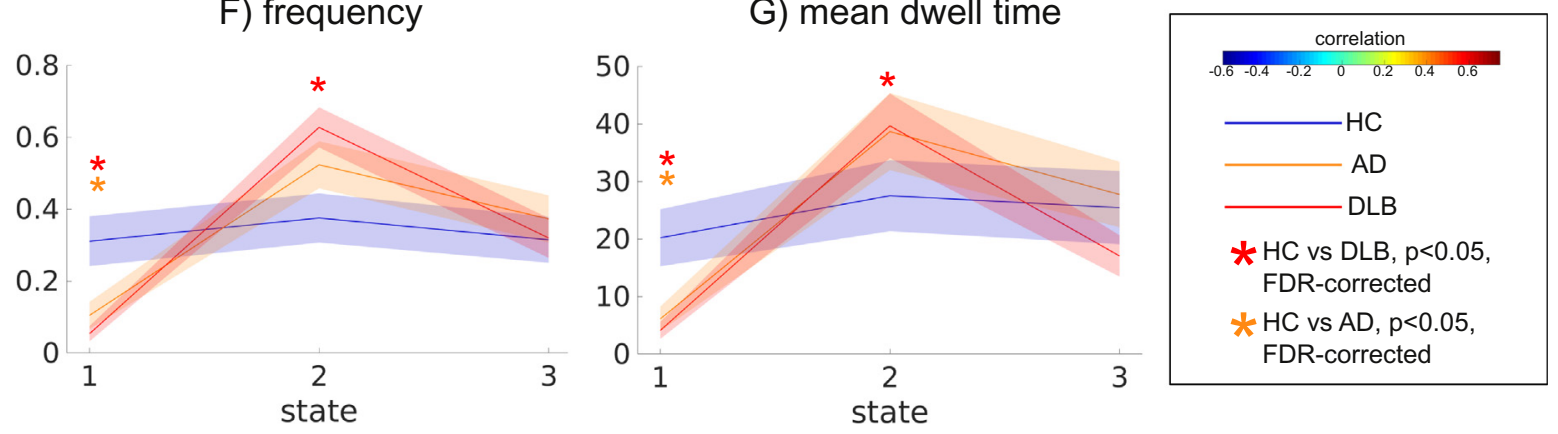

(caption on next page) 


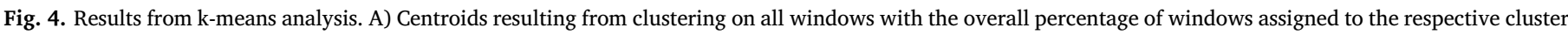

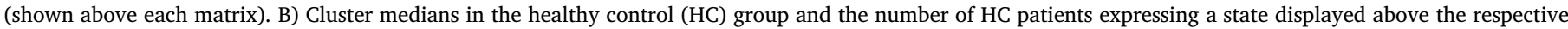

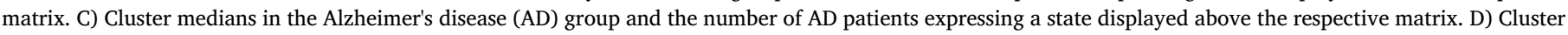

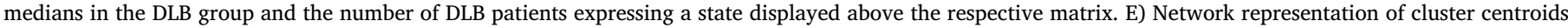

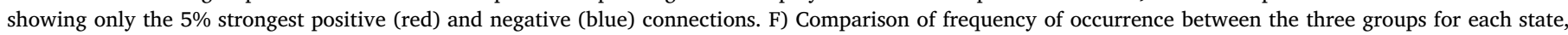

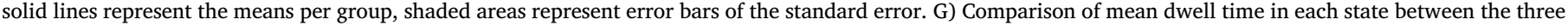
groups. FDR-corrected p-values $<0.05$ (from post-hoc tests) are marked with an asterisk.

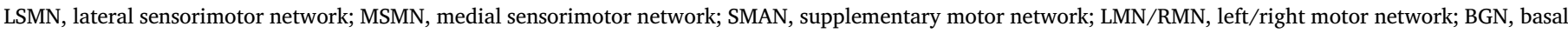

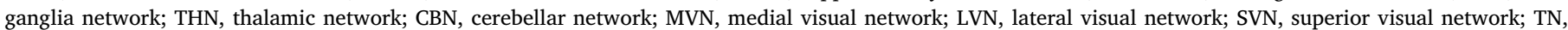

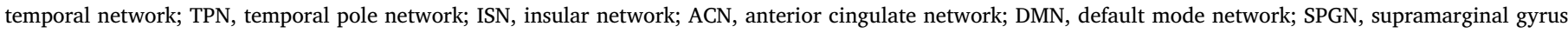
network; RFPN/LFPN, right/left fronto-parietal network; DAN, dorsal attention network; VAN, ventral attention network.
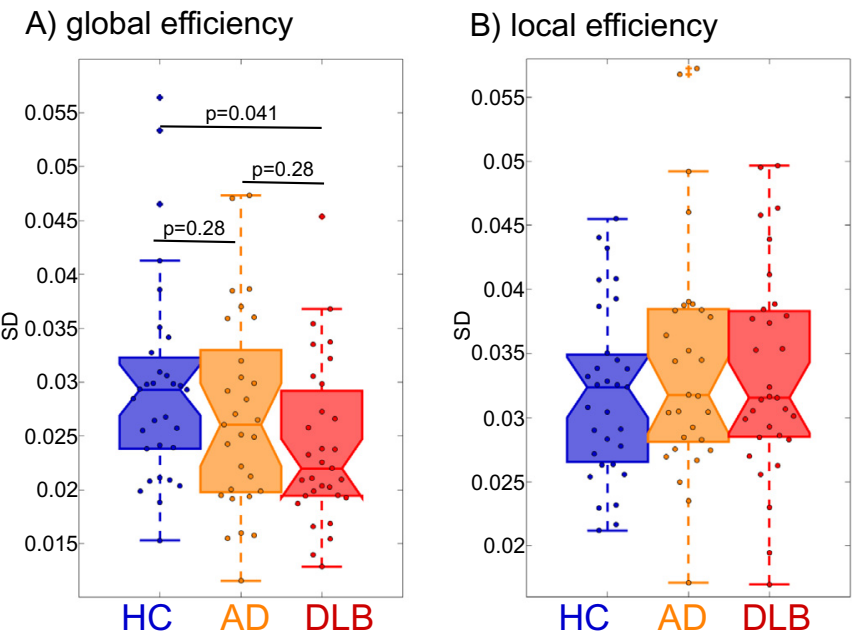

C) global efficiency for different densities

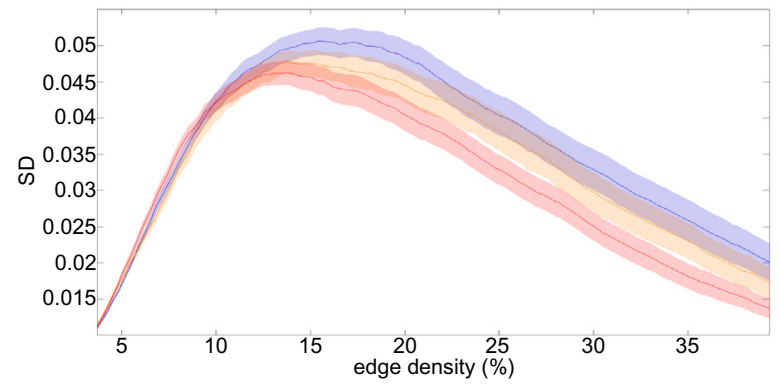

Fig. 5. Results from the dynamic network analysis. Comparison of the variability of A) global (p-values FDR-corrected) and B) local efficiency between groups. C) Variability of global efficiency at different network edge densities.

connectivity measures that showed group differences and mean framewise displacement (Supplementary Table S8).

\subsection{Effect of grey matter atrophy}

The AD group showed clusters of reduced GM volume compared to controls, mainly in right and left hippocampus (Supplementary Fig. S7). No regions showed increased GM volume in $\mathrm{AD}$ compared to controls. The DLB group had reduced GM volume in a small cluster in right cingulate cortex (Supplementary Fig. S8). Again, there were no areas of increased GM volume in DLB compared to controls. There were no significant differences in regional GM volume between the two dementia groups.

Mean GM volume (in litres) was $0.56(\mathrm{SD}=0.06)$ in healthy controls, $0.53(\mathrm{SD}=0.06)$ in $\mathrm{AD}$ patients, and $0.53(\mathrm{SD}=0.06)$ in the $\mathrm{LBD}$ group. Since this was not normally distributed in the AD group, a Kruskal-Wallis ANOVA was performed to compare total GM volume between the groups. This test revealed a marginally significant overall effect of group $\left(\mathrm{H}_{2}=6.09, \mathrm{p}=0.048\right)$. However, post-hoc Dunn's tests showed that there were no significant differences between any groups $(\mathrm{p}(\mathrm{HC}, \mathrm{AD})=0.06, \mathrm{p}(\mathrm{HC}, \mathrm{DLB})=0.18, \mathrm{p}(\mathrm{AD}, \mathrm{DLB})=1.0)$. The same analysis was repeated for TIV which did not show any differences between the groups (univariate ANOVA, $\mathrm{F}_{2,88}=0.23, \mathrm{p}=0.80$ ).

Adding total GM volume or TIV as covariates when residualizing the windowed correlation matrices had no effect on the estimation of standard deviation matrices or the cluster centroids resulting from the k-means analysis (see Supplementary Figs. S9 and S10).

\section{Discussion}

In this study we investigated differences in functional connectivity dynamics and dynamic brain network topology between patients with $\mathrm{DLB}$, patients with $\mathrm{AD}$, and healthy controls. In terms of dynamic changes in overall network structure, we found reduced variability of global efficiency in the DLB group compared to controls which was not observed in the AD group. Using a state-based analysis it became evident that both dementia groups spent less time in a state of strong internetwork connectivity than controls. Additionally, DLB patients spent more time in a more sparsely connected state characterized by the relative loss of strong anti-correlations and an isolation of motor networks relative to other networks. While dynamic connectivity measures of the $\mathrm{AD}$ group were often between those of the control and DLB groups, we did not see significant differences in the direct comparison of both dementia groups.

\subsection{State-based analysis}

While the number of states visited and the number of state changes was not altered in the dementia groups, there was a significant difference in the type of state changes in dementia patients compared to controls. The frequency with which the control participants visited each of the three states was relatively balanced, i.e. they spent about a third of their time in each state. In contrast, the distribution of states in the $\mathrm{AD}$ and $\mathrm{DLB}$ groups was more out of balance compared to controls with a clear decrease in frequency of state 1 in both dementia groups accompanied by an increased frequency of state 2 in DLB. In addition to visiting state 1 less often, the dementia patients also switched out of this state more rapidly and DLB patients stayed in state 2 for longer consecutive periods of time. In accordance with previous reports in healthy participants (Allen et al., 2014), development (Marusak et al., 2016), ageing (Viviano et al., 2017), and Parkinson's disease (PD) (Kim et al., 2017), the most common state in the present study (state 2) was characterized by a sparse connectivity profile with relatively weak inter-network connections and the absence of strong anti-correlations. The frequency of this state has been linked to the amount of self-focused thought (Marusak et al., 2016) and it has been suggested to represent a general connectivity pattern that participants spend most of their time in, with other states reflecting temporary deviations that might be due to cognitive, physiological, or motion-related processes (Viviano et al., 2017). State 1 deviated from this state by stronger positive and negative connections. It seems that $\mathrm{AD}$ as well as DLB patients remain in states of low inter-network connectivity and switch less often into more highly and specifically connected network configurations. This may relate to 
the presence of dementia in general even though we did not see any specific correlations between the time spent in different states and the severity of cognitive impairment. A specific hallmark of state 1 is strong connectivity within visual and motor networks and between these two groups of networks that is not present in state 2 . A reduced ability to switch into this state thus accords with Sourty et al. (2016) who found dynamic connectivity changes in DLB for networks related to visual processing using Hidden Markov Models. Another important characteristic of state 1 that differentiates it from state 2 is the existence of strong anti-correlations in the former. Furthermore, while the DMNs do not show strong correlations with task-positive networks in state 1, the other two states are characterized by positive connections between DMN, visual and attention networks. Anti-correlation between default mode and task-positive networks has been shown to be important for attentional function (Fox et al., 2005) and a loss of anti-correlations has been associated with ageing, mild cognitive impairment, and cognitive impairment in PD (Baggio et al., 2015; Esposito et al., 2017). Our results further suggest that an absence of this antithetic relationship between default mode and task-positive networks might also be a feature of more established neurodegenerative disease in the case of $\mathrm{AD}$ and DLB.

\subsection{DLB-related changes in dynamic network topology}

Regarding dynamic network topology, we found less variable global efficiency in DLB compared to controls. Global efficiency is a measure of communication efficiency across the whole brain network (Latora and Marchiori, 2001). In general, more pronounced variability of functional connectivity has been shown to be related to superior performance on a range of behavioral tests including attention and memory tasks (Jia et al., 2014) indicating that the dynamic and flexible engaging and disengaging of different brain regions seems to be crucial for efficient and adaptable communication within the brain (Zalesky et al., 2014). Reduced dynamics in turn can lead to less flexible, ineffective communication and a reduced ability of the network to respond to situational demands. The reduced variability of global efficiency in DLB might thus indicate a disease-related and abnormal rigidity of the brain network which might relate to the cognitive slowing (bradyphrenia) that is observed in DLB patients (Firbank et al., 2018). In contrast, in healthy brains efficiency is temporally modulated which has been shown to represent more economical network dynamics allowing for a more specific response to situational demands (Zalesky et al., 2014). In contrast to the dynamic analysis, we did not see significant group differences when considering efficiency across the whole time course. This finding stands in contrast to a previous study from our group which reported increased global efficiency in DLB compared to AD (Peraza et al., 2015). However, Peraza et al. (2015) performed a network analysis on the basis of atlas-defined regions of interest whereas network nodes in our analysis were RSNs. In both approaches the extraction of the time series is very different; in the case of network analysis based on RSNs, the efficiency of communication between whole-brain networks is assessed whereas Peraza et al. (2015) investigated the efficiency of communication between individual cortical regions. Nevertheless, similar to our results, Peraza et al. (2015) reported no difference between $\mathrm{AD}$ and controls with respect to global efficiency which indicates that static and dynamic changes in efficiency might be a specific feature of DLB that might not be associated with dementia per se. In contrast to our results, Kim et al. (2017) found increased variability of global efficiency in PD. However, this finding was not replicated in another study in PD patients with mild cognitive impairment (Díez-Cirarda et al., 2017) and thus further research will be needed to identify the specific changes related to these different Lewy body diseases.

\subsection{Relation to clinical symptoms in $D L B$}

Given the transient nature of clinical DLB symptoms such as visual hallucinations and cognitive fluctuations, we expected to find relations between symptom severity and dynamic connectivity measures. However, we did not observe any relation with respect to visual hallucinations and cognitive fluctuations, even before correcting for multiple comparisons. A possible reason for this might be the difference in time-scales: while our data only allowed the characterization of dynamics during a 6-minute resting state scan, the time-scale of cognitive fluctuations and visual hallucinations can be on the order of minutes to hours and even days. Performing repeated scans with DLB patients at different times of the day or over several days might thus help to understand more about the relation between functional connectivity dynamics and clinical symptom severity. There was a trend for an association between frequency of state 2 and severity of Parkinsonism in DLB, i.e. an increased time spent in this sparsely connected state might relate to more severe Parkinsonism. Relative to state 1 , this state was characterized by a disconnection of motor networks from other networks and the observed correlation might thus indicate that the isolation of motor networks might contribute to the severity of clinical motor symptoms. However, this is only an exploratory result that did not survive multiple comparison correction and further research will be needed to confirm this conjecture.

\subsection{Reliability of dynamic connectivity results}

The interpretation, functional significance, and origin of dynamic functional connectivity have been the subject of an extensive debate (Hindriks et al., 2016; Laumann et al., 2016). However, recent studies using concurrent fMRI and EEG measurements point towards a neuronal origin of dynamic functional connectivity (Chang et al., 2013). Additionally, several studies have provided support for a cognitive role by showing that temporary changes in connectivity are related to changes in behavioral or vigilance states (Jia et al., 2014; Kucyi et al., 2017; Thompson et al., 2013) and cognitive performance in healthy older adults (Cabral et al., 2017). Finally, the study of dynamic functional connectivity in clinical populations has led to the identification of specific dynamic connectivity alterations associated with specific disorders which provides further evidence of the neurocognitive significance of time-varying functional connectivity (Damaraju et al., 2014; Jones et al., 2012; Sourty et al., 2016).

Although the sliding window approach has been widely applied to study dynamic functional connectivity (Allen et al., 2014; Damaraju et al., 2014; Hutchison and Morton, 2015; Jones et al., 2012; Marusak et al., 2016) its validity has been debated (Hindriks et al., 2016). Advantages are its interpretability and computational efficiency which make this kind of analysis especially suitable for the investigation of clinical questions. However, problematic aspects include the need for an a priori specification of parameters such as window length and the number of states for the k-means analysis and the possibility of spurious connectivity fluctuations which can arise due to noise sources such as head motion (Hutchison et al., 2013a). In the present study we applied several pre- and postprocessing steps to reduce the effect of these noise sources (see Section 2.4). It was also ensured that the groups did not differ with respect to motion which makes it unlikely that the observed group differences were merely motion artefacts. Additionally, there was no significant relation between dynamic connectivity measures and mean framewise displacement indicating little influence of motion on the dynamic connectivity measures in our participants. Regarding the choice of window length, we showed that our results can be reproduced using windows of different lengths (see Supplementary Figs. S1 and S5). While most previous studies examined a larger number of states (Allen et al., 2014; Damaraju et al., 2014; Hutchison and Morton, 2015; Marusak et al., 2016; Viviano et al., 2017), we focused on a smaller set of three states which was determined as the optimal number of states in 
our dataset and is comparable to a previous report in PD (Kim et al., 2017). The states tended to get more unstable as more states were added with states appearing that were specific to certain participants (Supplementary Fig. S4). This might be due to the small number of participants and large heterogeneity in our sample. Nevertheless, we showed that the observed group differences in terms of frequency and dwell time remained largely unchanged for different values of $\mathrm{k}$ and states were reproducible on split-half and bootstrap resamples of the data which confirms the robustness of this approach. Notably, adding more states did not result in more significant group differences indicating that these three states represent the most important states in terms of dementia-related changes in connectivity dynamics.

A potential limitation in the interpretation of dynamic connectivity results is that dynamic connectivity measures are sensitive to changes in other features of the data such as variance and signal-to-noise ratio of the fMRI time series and non-stationarities in mean and variance. In theory, this problem can be overcome by generating data under the null hypothesis of static connectivity and showing that the probability of finding group differences in dynamic connectivity from these data is low. However, while for Gaussian data this can be achieved by using coherent phase randomization (Hindriks et al., 2016) or vector autoregressive null models (Zalesky et al., 2014), these techniques cannot be applied in the present context due to the non-Gaussianity of the RSN time series. This therefore remains as a potential limitation of this study.

Another factor that might influence dynamic connectivity measures is GM atrophy and group differences in GM atrophy have been shown to potentially lead to spurious results in a group comparison of functional connectivity (Damoiseaux et al., 2012). However, we confirmed that there were only subtle regional GM differences between the three groups included in this study and adding total GM volume as a covariate did not change the results.

\subsection{Limitations}

A limitation of this study is that over half of the DLB patients were on dopaminergic medication and scanned in the ON state which might have influenced their functional connectivity measures. However, dopaminergic medication has been shown to normalize connectivity towards more healthy levels (Tahmasian et al., 2015) suggesting that the observed group differences were not due to medication. Furthermore, we did not find differences in terms of dynamic connectivity measures between DLB patients who were taking dopaminergic medication compared to those not on these medications. All diagnoses were based on clinical assessment rather than pathological confirmation. However, the standardized diagnostic criteria that were used in this study have demonstrated high specificity when validated against autopsy findings (McKeith et al., 2000).

\subsection{Conclusion}

The loss of variability of global efficiency in DLB indicates an abnormally rigid brain network. This might be associated with less economical dynamics that can prevent specific and effective responses of the brain network to situational demands. This loss of dynamics was not observed in $\mathrm{AD}$ patients and seems to represent a DLB-specific abnormality that might relate to the cognitive slowing observed in DLB patients. In contrast, the inability to switch out of states of low internetwork connectivity into more highly and specifically connected network configurations was observed in both dementia groups and might thus be related to the presence of dementia in general rather than any symptoms that are specific to AD or DLB.

\section{Acknowledgements}

J.S. is supported by the Alzheimer's Society Doctoral Training
Centre at Newcastle University. M.K. is supported by the Engineering and Physical Sciences Research Council of the United Kingdom Grant EP/K026992/1. The research was supported by a Wellcome Trust Intermediate Clinical Fellowship (WT088441MA) to J.-P.T., Northumberland Tyne and Wear NHS Foundation Trust, by National Institute for Health Research (NIHR)Newcastle Biomedical Research Centre (BRC) based at Newcastle upon Tyne Hospitals NHS Foundation Trust and Newcastle University, and by Alzheimer's Research UK.

\section{Appendix A. Supplementary data}

Supplementary data to this article can be found online at https:// doi.org/10.1016/j.nicl.2019.101812.

\section{References}

Aarsland, D., Ballard, C.G., Larsen, J.P., McKeith, I.G., 2001. A comparative study of psychiatric symptoms in dementia with Lewy bodies and Parkinson's disease with and without dementia. Int. J. Geriatr. Psychiatry 16, 528-536. https://doi.org/10.1002/ gps.389.

Abou Elseoud, A., Littow, H., Remes, J.J., Starck, T., Nikkinen, J., Nissilä, J., Timonen, M., Tervonen, O., Kiviniemi, V., 2011. Group-ICA model order highlights patterns of functional brain connectivity. Front. Syst. Neurosci. 5 (37). https://doi.org/10.3389/ fnsys.2011.00037.

Achard, S., Bullmore, E., 2007. Efficiency and cost of economical brain functional networks. PLoS Comput. Biol. 3, 0174-0183. https://doi.org/10.1371/journal.pcbi. 0030017.

Agosta, F., Pievani, M., Geroldi, C., Copetti, M., Frisoni, G.B., Filippi, M., 2012. Resting state fMRI in Alzheimer's disease: beyond the default mode network. Neurobiol. Aging 33, 1564-1578. https://doi.org/10.1016/j.neurobiolaging.2011.06.007.

Allen, E.A., Damaraju, E., Plis, S.M., Erhardt, E.B., Eichele, T., Calhoun, V.D., 2014. Tracking whole-brain connectivity dynamics in the resting state. Cereb. Cortex 24, 663-676. https://doi.org/10.1093/cercor/bhs352.

Avants, B.B., Tustison, N.J., Song, G., Cook, P.A., Klein, A., Gee, J.C., 2011. A reproducible evaluation of ANTs similarity metric performance in brain image registration. Neuroimage 54, 2033-2044. https://doi.org/10.1016/j.neuroimage.2010. 09.025 .

Baggio, H.-C., Segura, B., Sala-Llonch, R., Marti, M.-J., Valldeoriola, F., Compta, Y., Tolosa, E., Junqué, C., 2015. Cognitive impairment and resting-state network connectivity in Parkinson's disease. Hum. Brain Mapp. 36, 199-212. https://doi.org/10 1002/hbm.22622.

Beckmann, C.F., DeLuca, M., Devlin, J.T., Smith, S.M., 2005. Investigations into restingstate connectivity using independent component analysis. Philos. Trans. R. Soc. Lond. Ser. B Biol. Sci. 360, 1001-1013. https://doi.org/10.1098/rstb.2005.1634.

Biswal, B.B., Mennes, M., Zuo, X.-N., Gohel, S., Kelly, C., Smith, S.M., Beckmann, C.F., Adelstein, J.S., Buckner, R.L., Colcombe, S., Dogonowski, A.-M., Ernst, M., Fair, D., Hampson, M., Hoptman, M.J., Hyde, J.S., Kiviniemi, V.J., Kotter, R., Li, S.-J., Lin, C.P., Lowe, M.J., Mackay, C., Madden, D.J., Madsen, K.H., Margulies, D.S., Mayberg, H.S., McMahon, K., Monk, C.S., Mostofsky, S.H., Nagel, B.J., Pekar, J.J., Peltier, S.J., Petersen, S.E., Riedl, V., Rombouts, S.A.R.B., Rypma, B., Schlaggar, B.L., Schmidt, S., Seidler, R.D., Siegle, G.J., Sorg, C., Teng, G.-J., Veijola, J., Villringer, A., Walter, M., Wang, L., Weng, X.-C., Whitfield-Gabrieli, S., Williamson, P., Windischberger, C., Zang, Y.-F., Zhang, H.-Y., Castellanos, F.X., Milham, M.P., 2010. Toward discovery science of human brain function. Proc. Natl. Acad. Sci. 107, 4734-4739. https://doi. org/10.1073/pnas.0911855107.

Bradshaw, J., Saling, M., Hopwood, M., Anderson, V., Brodtmann, A., 2004. Fluctuating cognition in dementia with Lewy bodies and Alzheimer's disease is qualitatively distinct. J. Neurol. Neurosurg. Psychiatry 75, 382-387. https://doi.org/10.1136/ jnnp.2002.002576.

Burchett, W.W., Ellis, A.R., Harrar, S.W., Bathke, A.C., 2017. Nonparametric inference for multivariate data: the R package npmv. J. Stat. Softw. 76. https://doi.org/10.18637/ jss.v076.i04.

Cabral, J., Vidaurre, D., Marques, P., Magalhães, R., Silva Moreira, P., Miguel Soares, J., Deco, G., Sousa, N., Kringelbach, M.L., 2017. Cognitive performance in healthy older adults relates to spontaneous switching between states of functional connectivity during rest. Sci. Rep. 7 (5135). https://doi.org/10.1038/s41598-017-05425-7.

Calhoun, V.D., Miller, R.L., Pearlson, G.D., Adali, T., 2014. The Chronnectome: timevarying connectivity networks as the next frontier in fMRI data discovery. Neuron 84 262-274. https://doi.org/10.1016/j.neuron.2014.10.015.

Cerliani, L., Mennes, M., Thomas, R.M., Di Martino, A., Thioux, M., Keysers, C., 2015 Increased functional connectivity between subcortical and cortical resting-state networks in autism Spectrum disorder. JAMA Psychiatry 72, 767. https://doi.org/10. 1001/jamapsychiatry.2015.0101.

Chang, C., Glover, G.H., 2010. Time-frequency dynamics of resting-state brain connectivity measured with fMRI. Neuroimage 50, 81-98. https://doi.org/10.1016/j. neuroimage.2009.12.011.

Chang, C., Liu, Z., Chen, M.C., Liu, X., Duyn, J.H., 2013. EEG correlates of time-varying BOLD functional connectivity. Neuroimage 72, 227-236. https://doi.org/10.1016/j. neuroimage.2013.01.049.

Cummings, J.L., Mega, M., Gray, K., Rosenberg-Thompson, S., Carusi, D.A., Gornbein, J., 1994. The neuropsychiatric inventory: comprehensive assessment of 
psychopathology in dementia. Neurology 44, 2308-2314. https://doi.org/10.1212/ WNL.44.12.2308.

Damaraju, E., Allen, E.A., Belger, A., Ford, J.M., McEwen, S., Mathalon, D.H., Mueller, B.A., Pearlson, G.D., Potkin, S.G., Preda, A., Turner, J.A., Vaidya, J.G., van Erp, T.G. Calhoun, V.D., 2014. Dynamic functional connectivity analysis reveals transient states of dysconnectivity in schizophrenia. NeuroImage Clin. 5, 298-308. https://doi. org/10.1016/j.nicl.2014.07.003.

Damoiseaux, J.S., Prater, K.E., Miller, B.L., Greicius, M.D., 2012. Functional connectivity tracks clinical deterioration in Alzheimer's disease. Neurobiol. Aging 33, 828.e19-828.e30. https://doi.org/10.1016/j.neurobiolaging.2011.06.024.

Díez-Cirarda, M., Strafella, A.P., Kim, J., Peña, J., Ojeda, N., Cabrera-Zubizarreta, A., Ibarretxe-Bilbao, N., 2017. Dynamic functional connectivity in Parkinson's disease patients with mild cognitive impairment and normal cognition. NeuroImage Clin. https://doi.org/10.1016/j.nicl.2017.12.013.

Esposito, R., Cieri, F., Chiacchiaretta, P., Cera, N., Lauriola, M., Di Giannantonio, M., Tartaro, A., Ferretti, A., 2017. Modifications in resting state functional anticorrelation between default mode network and dorsal attention network: comparison among young adults, healthy elders and mild cognitive impairment patients. Brain Imaging Behav. 1-15. https://doi.org/10.1007/s11682-017-9686-y.

Fahn, S., Elton, R., 1987. Members of the UPDRS development committee. Recent Dev. Park. Dis. 2, 153

Firbank, M.J., O'Brien, J.T., Taylor, J.P., 2018. Long reaction times are associated with delayed brain activity in lewy body dementia. Hum. Brain Mapp. 39, 633-643. https://doi.org/10.1002/hbm.23866.

Fox, M.D., Snyder, A.Z., Vincent, J.L., Corbetta, M., Van Essen, D.C., Raichle, M.E., 2005. The human brain is intrinsically organized into dynamic, anticorrelated functional networks. Proc. Natl. Acad. Sci. 102, 9673-9678. https://doi.org/10.1073/pnas. 0504136102.

Hindriks, R., Adhikari, M.H., Murayama, Y., Ganzetti, M., Mantini, D., Logothetis, N.K., Deco, G., 2016. Can sliding-window correlations reveal dynamic functional connectivity in resting-state fMRI? Neuroimage 127, 242-256. https://doi.org/10.1016/ j.neuroimage.2015.11.055.

Hutchison, R.M., Morton, J.B., 2015. Tracking the Brain's functional coupling dynamics over development. J. Neurosci. 35, 6849-6859. https://doi.org/10.1523/ JNEUROSCI.4638-14.2015.

Hutchison, R.M., Womelsdorf, T., Allen, E.A., Bandettini, P.A., Calhoun, V.D., Corbetta M., Della Penna, S., Duyn, J.H., Glover, G.H., Gonzalez-Castillo, J., Handwerker, D.A., Keilholz, S.D., Kiviniemi, V., Leopold, D.A., de Pasquale, F., Sporns, O., Walter, M., Chang, C., 2013a. Dynamic functional connectivity: promise, issues, and interpretations. Neuroimage 80, 360-378. https://doi.org/10.1016/j.neuroimage.2013. 05.079.

Hutchison, R.M., Womelsdorf, T., Gati, J.S., Everling, S., Menon, R.S., 2013b. Restingstate networks show dynamic functional connectivity in awake humans and anesthetized macaques. Hum. Brain Mapp. 34, 2154-2177. https://doi.org/10.1002/ hbm. 22058.

Jia, H., Hu, X., Deshpande, G., 2014. Behavioral relevance of the dynamics of the functional brain connectome. Brain Connect. 4, 741-759. https://doi.org/10.1089/brain 2014.0300.

Jones, D.T., Vemuri, P., Murphy, M.C., Gunter, J.L., Senjem, M.L., Machulda, M.M., Przybelski, S.A., Gregg, B.E., Kantarci, K., Knopman, D.S., Boeve, B.F., Petersen, R.C., Jr, C.R.J., 2012. Non-stationarity in the "resting Brain's" modular architecture. PLoS Biol. 7, e39731. https://doi.org/10.1371/journal.pone.0039731.

Kelly, R.E., Alexopoulos, G.S., Wang, Z., Gunning, F.M., Murphy, C.F., Morimoto, S.S., Kanellopoulos, D., Jia, Z., Lim, K.O., Hoptman, M.J., 2010. Visual inspection of independent components: defining a procedure for artifact removal from fMRI data. J. Neurosci. Methods 189, 233-245. https://doi.org/10.1016/j.jneumeth.2010.03.028.

Kenny, E.R., Blamire, A.M., Firbank, M.J., O'Brien, J.T., 2012. Functional connectivity in cortical regions in dementia with Lewy bodies and Alzheimer's disease. Brain 135, 569-581. https://doi.org/10.1093/brain/awr327.

Kim, J., Criaud, M., Cho, S.S., Díez-Cirarda, M., Mihaescu, A., Coakeley, S., Ghadery, C., Valli, M., Jacobs, M.F., Houle, S., Strafella, A.P., 2017. Abnormal intrinsic brain functional network dynamics in Parkinson's disease. Brain 140, 2955-2967. https:// doi.org/10.1093/brain/awx233.

Kucyi, A., Hove, M.J., Esterman, M., Hutchison, R.M., Valera, E.M., 2017. Dynamic brain network correlates of spontaneous fluctuations in attention. Cereb. Cortex 27, 1831-1840. https://doi.org/10.1093/cercor/bhw029.

Latora, V., Marchiori, M., 2001. Efficient behavior of small-world networks. Phys. Rev. Lett. 87, 198701. https://doi.org/10.1103/PhysRevLett.87.198701.

Laumann, T.O., Snyder, A.Z., Mitra, A., Gordon, E.M., Gratton, C., Adeyemo, B., Gilmore, A.W., Nelson, S.M., Berg, J.J., Greene, D.J., McCarthy, J.E., Tagliazucchi, E., Laufs, H., Schlaggar, B.L., Dosenbach, N.U.F., Petersen, S.E., 2016. On the stability of BOLD fMRI correlations. Cereb. Cortex 27, 4719-4732. https://doi.org/10.1093/cercor/ bhw265.

Liao, W., Chen, H., Feng, Y., Mantini, D., Gentili, C., Pan, Z., Ding, J., Duan, X., Qiu, C., Lui, S., Gong, Q., Zhang, W., 2010. Selective aberrant functional connectivity of resting state networks in social anxiety disorder. Neuroimage 52, 1549-1558. https://doi.org/10.1016/j.neuroimage.2010.05.010.

Lowther, E.R., O'Brien, J.T., Firbank, M.J., Blamire, A.M., 2014. Lewy body compared with Alzheimer dementia is associated with decreased functional connectivity in resting state networks. Psychiatry Res. Neuroimaging 223, 192-201. https://doi.org/ 10.1016/j.pscychresns.2014.06.004.
Marusak, H.A., Calhoun, V.D., Brown, S., Crespo, L.M., Sala-Hamrick, K., Gotlib, I.H., Thomason, M.E., 2016. Dynamic functional connectivity of neurocognitive networks in children. Hum. Brain Mapp. 00, 1-12. https://doi.org/10.1002/hbm.23346.

McKeith, I.G., Ballard, C.G., Perry, R.H., Ince, P.G., O'Brien, J.T., Neill, D., Lowery, K. Jaros, E., Barber, R., Thompson, P., Swann, A., Fairbairn, a F., Perry, E.K., 2000. Prospective validation of Consensus criteria for the diagnosis of dementia with Lewy bodies. Neurology 54, 1050-1058. https://doi.org/10.1212/WNL.54.5.1050.

McKeith, I.G., Dickson, D.W., Lowe, J., Emre, M., O'Brien, J.T., Feldman, H., Cummings, J., Duda, J.E., Lippa, C., Perry, E.K., Aarsland, D., Arai, H., Ballard, C.G., Boeve, B., Burn, D.J., Costa, D., Del Ser, T., Dubois, B., Galasko, D., Gauthier, S., Goetz, C.G., Gomez-Tortosa, E., Halliday, G., Hansen, L.A., Hardy, J., Iwatsubo, T., Kalaria, R.N., Kaufer, D., Kenny, R.A., Korczyn, A., Kosaka, K., Lee, V.M.Y., Lees, A., Litvan, I., Londos, E., Lopez, O.L., Minoshima, S., Mizuno, Y., Molina, J.A., MukaetovaLadinska, E.B., Pasquier, F., Perry, R.H., Schulz, J.B., Trojanowski, J.Q., Yamada, M., 2005. Diagnosis and management of dementia with Lewy bodies: third report of the DLB consortium. Neurology 65, 1863-1872. https://doi.org/10.1212/01.wnl. 0000187889.17253.b1.

McKhann, G., Drachman, D., Folstein, M., Katzman, R., 1984. Clinical diagnosis of Alzheimer's disease: report of the NINCDS- ADRDA work group under the auspices of Department of Health and Human Services Task Force on Alzheimer's disease. Neurology 34, 939-944. https://doi.org/10.3233/JAD-122299.

McKhann, G.M., Knopman, D.S., Chertkow, H., Hyman, B.T., Jack, C.R., Kawas, C.H., Klunk, W.E., Koroshetz, W.J., Manly, J.J., Mayeux, R., Mohs, R.C., Morris, J.C., Rossor, M.N., Scheltens, P., Carrillo, M.C., Thies, B., Weintraub, S., Phelps, C.H. 2011. The diagnosis of dementia due to Alzheimer's disease: recommendations from the National Institute on Aging-Alzheimer's Association workgroups on diagnostic guidelines for Alzheimer's disease. Alzheimers Dement. 7, 263-269. https://doi.org/ 10.1016/j.jalz.2011.03.005.

Peraza, L.R., Kaiser, M., Firbank, M.J., Graziadio, S., Bonanni, L., Onofrj, M., Colloby, S.J., Blamire, A.M., O'Brien, J.T., Taylor, J.-P., 2014. fMRI resting state networks and their association with cognitive fluctuations in dementia with Lewy bodies. NeuroImage. Clin. 4, 558-565. https://doi.org/10.1016/j.nicl.2014.03.013.

Peraza, L.R., Taylor, J.-P., Kaiser, M., 2015. Divergent brain functional network alterations in dementia with Lewy bodies and Alzheimer's disease. Neurobiol. Aging 36, 2458-2467. https://doi.org/10.1016/j.neurobiolaging.2015.05.015.

Poppe, A.B., Wisner, K., Atluri, G., Lim, K.O., Kumar, V., MacDonald, A.W., 2013. Toward a neurometric foundation for probabilistic independent component analysis of fMRI data. Cogn. Affect. Behav. Neurosci. 13, 641-659. https://doi.org/10.3758/s13415013-0180-8.

Power, J.D., Barnes, K.A., Snyder, A.Z., Schlaggar, B.L., Petersen, S.E., 2012. Spurious but systematic correlations in functional connectivity MRI networks arise from subject motion. Neuroimage 59, 2142-2154. https://doi.org/10.1016/j.neuroimage.2011. 10.018 .

Pruim, R.H.R., Mennes, M., van Rooij, D., Llera, A., Buitelaar, J.K., Beckmann, C.F., 2015 ICA-AROMA: a robust ICA-based strategy for removing motion artifacts from fMRI data. Neuroimage 112, 267-277. https://doi.org/10.1016/j.neuroimage.2015.02. 064.

Rubinov, M., Sporns, O., 2010. Complex network measures of brain connectivity: uses and interpretations. Neuroimage 52, 1059-1069. https://doi.org/10.1016/j.neuroimage. 2009.10.003.

Schumacher, J., Peraza, L.R., Firbank, M.J., Thomas, A.J., Kaiser, M., Gallagher, P., O'Brien, J.T., Blamire, A.M., Taylor, J.-P., 2018. Functional connectivity in dementia with Lewy bodies: a within- and between-network analysis. Hum. Brain Mapp. 39, 1118-1129. https://doi.org/10.1002/hbm.23901.

Sourty, M., Thoraval, L., Roquet, D., Armspach, J.-P., Foucher, J., Blanc, F., 2016 Identifying dynamic functional connectivity changes in dementia with Lewy bodies based on product hidden Markov models. Front. Comput. Neurosci. 10 (60). https:// doi.org/10.3389/fncom.2016.00060.

Tahmasian, M., Bettray, L.M., van Eimeren, T., Drzezga, A., Timmermann, L., Eickhoff, C.R., Eickhoff, S.B., Eggers, C., 2015. A systematic review on the applications of resting-state fMRI in Parkinson's disease: does dopamine replacement therapy play a role? Cortex 73, 80-105. https://doi.org/10.1016/j.cortex.2015.08.005.

Thompson, G.J., Magnuson, M.E., Merritt, M.D., Schwarb, H., Pan, W.J., Mckinley, A., Tripp, L.D., Schumacher, E.H., Keilholz, S.D., 2013. Short-time windows of correlation between large-scale functional brain networks predict vigilance intraindividually and interindividually. Hum. Brain Mapp. 34, 3280-3298. https://doi.org/10.1002/ hbm. 22140 .

van Wijk, B.C.M., Stam, C.J., Daffertshofer, A., 2010. Comparing brain networks of different size and connectivity density using graph theory. PLoS ONE 5. https://doi.org/ 10.1371/journal.pone.0013701.

Viviano, R.P., Raz, N., Yuan, P., Damoiseaux, J.S., 2017. Associations between dynamic functional connectivity and age, metabolic risk, and cognitive performance. Neurobiol. Aging 59, 135-143. https://doi.org/10.1016/j.neurobiolaging.2017.08. 003

Walker, M.P., Ayre, G.A., Cummings, J.L., Wesnes, K.A., McKeith, I.G., O'Brien, J.T., Ballard, C.G., 2000. The clinician assessment of fluctuation and the one day fluctuation assessment scale. Br. J. Psychiatry 177, 252-256. https://doi.org/10.1192/ bjp.177.3.252.

Zalesky, A., Fornito, A., Cocchi, L., Gollo, L.L., Breakspear, M., 2014. Time-resolved resting-state brain networks. Proc. Natl. Acad. Sci. 111, 10341-10346. https://doi. org/10.1073/pnas.1400181111. 